\title{
Editorial
}

\section{Mathematical Aspects of Meshless Methods}

\author{
Yumin Cheng, ${ }^{1}$ Wenqing Wang, ${ }^{2}$ Miaojuan Peng, ${ }^{3}$ and Zan Zhang ${ }^{4}$ \\ ${ }^{1}$ Shanghai Institute of Applied Mathematics and Mechanics, Shanghai University, Shanghai 200072, China \\ ${ }^{2}$ Helmholtz Centre for Environmental Research-UFZ, Permoserstraße 15, 04318 Leipzig, Germany \\ ${ }^{3}$ Department of Civil Engineering, Shanghai University, Shanghai 200072, China \\ ${ }^{4}$ School of Science, East China University of Science and Technology, Shanghai 200237, China
}

Correspondence should be addressed to Yumin Cheng; ymcheng@shu.edu.cn

Received 28 May 2014; Accepted 28 May 2014; Published 5 June 2014

Copyright (c) 2014 Yumin Cheng et al. This is an open access article distributed under the Creative Commons Attribution License, which permits unrestricted use, distribution, and reproduction in any medium, provided the original work is properly cited.

Different from the conventional numerical methods, such as finite element method (FEM) and boundary element method (BEM), the meshless method is an approximation based on the nodes in the domain and it does not require a mesh to connect nodes for the solution of a problem. The meshless method can be applied to solve many complicated science and engineering problems, such as extremely large deformation and crack growth problems, which are not suitable to be solved by the conventional numerical methods [1].

The meshless method has been developed rapidly in recent 20 years. The development yields different types of meshless method, such as smoothed particle hydrodynamics (SPH) method [2], diffuse element method (DEM) [3], element-free Galerkin (EFG) method [4, 5], reproducing kernel particle method (RKPM) [6, 7], radial basis function (RBF) method $[8,9]$, finite point method (FPM) [10], meshless local Petrov-Galerkin (MLPG) method [11], complex variable element-free Galerkin (CVEFG) method [12-14], meshless manifold method $[15,16]$, mesh-free reproducing kernel particle Ritz method [17], boundary element-free method (BEFM) [18-20], local boundary integral equation (LBIE) method [21, 22], boundary node method (BNM) [23], and some others may not be mentioned here.

For a numerical method, the corresponding mathematical theory is very important. Without a decent mathematical theory background, the numerical method cannot be developed further. For many meshless methods, the corresponding mathematical theories are more complicated than that of the FEM and BEM due to its complicated shape functions. Except the radial basis function method, a few papers are published for the mathematical theories of other meshless methods, especially the EFG method, the RKPM, and the MLPG method, which have been studied and applied in many fields of science and engineering.

Levin obtained the error estimates of the moving leastsquares (MLS) approximation in the uniform norm of a regular function in high dimensions [24]. Under appropriate hypotheses on the weight function and the distribution of nodes, Armentano obtained the error estimates of the MLS approximation in one-dimensional case [25]. Zuppa also obtained error estimates for the MLS approximation and its derivatives by introducing condition numbers of the star of nodes in the normal equation [26]. R. Cheng and Y. Cheng studied the error estimates of the MLS approximation in multiple dimensions of Sobolev space [27]. And Li and Zhu also studied the error estimates of the MLS approximation for one-dimensional problems in Sobolev space [28]. Ren et al. discussed the complex variable interpolating moving least-squares method and the interpolating property [29]. Gavete et al. [30] and Rossi and Alves [31] studied the error estimates of the EFG method based on the MLS approximation. Moreover, R.-J. Cheng and Y.-M. Cheng discussed the error estimates of the EFG method for potential and elasticity problems $[32,33]$.

This special issue particularly takes an interest in manuscripts that report relevance of meshless methods in 
theory and applications. The special issue is published to summarize the most recent developments in meshless methods within the last five years, and it especially focuses on new methods and their corresponding mathematical theories. Moreover, the articles on the applications of meshless methods for solving complicated engineering problems are also included. Topics that have been addressed in this special issue cover

(i) mathematical theories of meshless methods,

(ii) approximation function of new meshless methods,

(iii) improvements of meshless methods,

(iv) simulations for complicated engineering problems.

About 20 manuscripts were submitted to this special issue for review, in which 14 manuscripts were accepted for publication, 5 manuscripts were rejected, and one manuscript was withdrawn by the corresponding author because of some mistakes found by the author himself.

In the article titled "The error estimates of the interpolating element-free Galerkin method for two-point boundary value problems" by J. F. Wang et al., a simpler formula of the shape function of the interpolating moving least-squares (IMLS) method is obtained. Then based on the IMLS method and the Galerkin weak form, an interpolating element-free Galerkin (IEFG) method for two-point boundary value problems is presented. And the IEFG method has high computing speed and precision. The error analysis of the IEFG method for twopoint boundary value problems is given. And the convergence rates of the numerical solution and its derivatives of the IEFG method are discussed.

In the article titled "The interpolating boundary elementfree method for unilateral problems arising in variational inequalities" by F. Li and X. Li, the interpolating boundary element-free method (IBEFM) is developed for boundaryonly analysis of unilateral problems. The IBEFM is a direct boundary-only meshless method that combines the improved IMLS method with boundary integral equations (BIE) [34]. And the convergence of the IBEFM is discussed mathematically.

The article titled "A modified SPH method for dynamic failure simulation of heterogeneous material" by G. W. Ma et al. is the application of the modified SPH method. An elastoplastic damage model based on an extension form of the unified twin shear strength criterion is adopted; polycrystalline modeling is introduced to generate the artificial microstructure of specimen for the dynamic simulation of Brazilian splitting test and uniaxial compression test, and the strain rate effect on the predicted dynamic tensile and compressive strength is discussed. The final failure patterns and the dynamic strength increments demonstrate good agreements with experimental results.

The article titled "Stress intensity factor for interface cracks in bimaterials using complex variable meshless manifold method" by H. Gao and G. Wei is the application of the complex variable meshless manifold method (CVMMM) to interface cracks between dissimilar materials. A discontinuous function and the near-tip asymptotic displacement functions are introduced into the complex variable moving least-squares (CVMLS) approximation, which is used to obtain the shape function. Numerical examples of bimaterial interfacial cracks are solved with the CVMMM to obtain the complex stress intensity factors.

In the article titled "Particle discrete method based on manifold cover for crack propagation of jointed rock mass" by Y. Ping et al., based on the particle contact model and the concept of manifold cover, the manifold particle discrete (MPD) method is presented. The MPD method can easily simulate the crack generation, propagation, and coalescence of jointed rock mass.

In the article titled "The incremental hybrid natural element method for elastoplasticity problems" by Y. Ma et al., an incremental hybrid natural element method (HNEM) is proposed. By introducing the concept of the hybrid stress element into the natural element method and using the incremental Hellinger-Reissner variational principle to obtain the system equations, the corresponding formulae of the HNEM for elastoplasticity problems are obtained. In the HNEM, the stress and displacement at each node can be obtained directly. Numerical examples are given to show that the solutions of the HNEM for the elastoplastic problems have higher precision than those of the NEM.

In the article titled "Geometric nonlinear meshless analysis of ribbed rectangular plates based on the FSDT and the moving least-squares approximation" by L. X. Peng et al., the plate and the ribs are considered separately, the approximation functions of the displacement, the stress and strain of the plate and the ribs are obtained using the MLS approximation, the virtual strain energy formulation of the plate and the ribs are derived separately, and the nonlinear equilibrium equation of the entire ribbed plate is given by the virtual work principle.

In the article titled "Analysis of the boundary knot method for 3D Helmholtz-type equation" by F. Z. Wang and K. H. Zheng, the regularization techniques and the effective condition number are introduced into boundary knot method (BKM) for the 3D Helmholtz-type problems to obtain the stable and convergent solutions. Numerical examples considering noisy and noise-free boundary conditions are given to show that the BKM in this article can obtain the stable numerical solutions.

In the article titled "The application of mesh-free method in the numerical simulation of beams with the size effect" by Y. Tian et al., the MLS approximation is used to construct the shape function, the intrinsic bulk length and the directional surface length components are introduced into the constitutive relationship to describe the size effect, and the variation of the total potential is provided to obtain the system equations. In the meshless method, the higher-order strains are directly approximated with the nodal components due to the higher-order continuity of the shape function. The convergence of the method is illustrated, and the effects of the intrinsic bulk length and the directional surface length components are studied.

In the article titled "The interpolating element-free Galerkin method for 2D transient heat conduction problems" by $\mathrm{N}$. Zhao and H. Ren, the shape function is constructed by the IMLS method, and Galerkin weak form is used to obtain 
the system equations, and then the interpolating elementfree Galerkin (IEFG) method for transient heat conduction problems is presented. The advantage of the IEFG method is that the essential boundary conditions can be applied directly.

In the article titled "The improved moving least-square Ritz method for the one-dimensional sine-Gordon equation" by Q. Wei and R. Cheng, the improved moving least-square approximation is employed to obtain the shape function of the $1 \mathrm{D}$ displacement field. The discrete system equations are obtained with the Ritz minimization procedure. Numerical examples of the sine-Gordon equation are solved to show the effectiveness and accuracy of the method in this article.

In the article titled "An improved interpolating elementfree Galerkin method based on nonsingular weight functions" by F. X. Sun et al., an improved interpolating moving least-squares (IIMLS) method based on nonsingular weight functions is discussed first. In the IIMLS method, the shape function satisfies the property of Kronecker $\delta$ function. Using the IIMLS method to obtain the shape function, an improved interpolating element-free Galerkin (IIEFG) method is presented for two-dimensional potential problems. In the IIEFG method, the essential boundary conditions are applied directly, and the number of unknown coefficients in the trial function of the IIMLS method is less than that of the MLS approximation, and then, under the same node distribution, the IIEFG method has higher computational precision than the EFG method and the IEFG method based on the singular weight functions.

In the article titled "A highly accurate regular domain collocation method for solving potential problems in the irregular doubly connected domains" by Z.-Q. Wang et al., a highly accurate regular domain collocation method is proposed. The formulae of the method are constructed by using barycentric Lagrange interpolation collocation method in the regular domain in polar coordinate system. Some numerical examples are given to demonstrate the effectiveness and accuracy of the method in this article.

The article titled "Eigenstrain boundary integral equations with local Eshelby matrix for stress analysis of ellipsoidal particles" by H. Ma et al. is for the large scale numerical simulation of particle reinforced materials. The concept of local Eshelby matrix is introduced into the computational model of the eigenstrain BIE to solve the problem of interactions among particles. Three-dimensional stress analyses are carried out for some ellipsoidal particles with various Young's modulus ratios and different shapes to verify the feasibility and efficiency of the improved eigenstrain BIE algorithm.

\section{Acknowledgments}

We thank all authors for their new research work submitted to this special issue of mathematical problems in engineering. And we are also very grateful to the reviewers for their professional comments for the manuscripts.

Yumin Cheng Wenqing Wang Miaojuan Peng Zan Zhang

\section{References}

[1] D. M. Li, K. M. Liew, and Y. M. Cheng, "An improved complex variable element-free Galerkin method for two-dimensional large deformation elastoplasticity problems," Computer Methods in Applied Mechanics and Engineering, vol. 269, pp. 72-86, 2014.

[2] R. A. Gingold and J. J. Monaghan, "Smoothed particle hydrodynamics: theory and allocation to non-spherical stars," Monthly Notices of the Royal Astronomical Society, vol. 181, pp. 375-389, 1977.

[3] B. Nayroles, G. Touzot, and P. Villon, "Generalizing the finite element method: diffuse approximation and diffuse elements," Computational Mechanics, vol. 10, no. 5, pp. 307-318, 1992.

[4] T. Belytschko, Y. Y. Lu, and L. Gu, "Element-free Galerkin methods," International Journal for Numerical Methods in Engineering, vol. 37, no. 2, pp. 229-256, 1994.

[5] Z. Zhang, J. Wang, Y. Cheng, and K. M. Liew, “The improved element-free Galerkin method for three-dimensional transient heat conduction problems," Science China Physics, Mechanics and Astronomy, vol. 56, no. 8, pp. 1568-1580, 2013.

[6] W. K. Liu, S. Jun, and Y. F. Zhang, "Reproducing kernel particle methods," International Journal for Numerical Metheds in Engineering, vol. 20, no. 8-9, pp. 1081-1106, 1995.

[7] L. Chen and Y. M. Cheng, "The complex variable reproducing kernel particle method for elasto-plasticity problems," Science China Physics, Mechanics and Astronomy, vol. 53, no. 5, pp. 954965, 2010.

[8] Z.-M. Wu and R. Schaback, "Local error estimates for radial basis function interpolation of scattered data," IMA Journal of Numerical Analysis, vol. 13, no. 1, pp. 13-27, 1993.

[9] B.-D. Dai and Y.-M. Cheng, "Local boundary integral equation method based on radial basis functions for potential problems," Acta Physica Sinica, vol. 56, no. 2, pp. 597-603, 2007.

[10] E. Oñate, S. Idelsohn, O. C. Zienkiewicz, and R. L. Taylor, "A finite point method in computational mechanics. Applications to convective transport and fluid flow," International Journal for Numerical Methods in Engineering, vol. 39, no. 22, pp. 3839$3866,1996$.

[11] S. N. Atluri and T. Zhu, "A new Meshless Local Petrov-Galerkin (MLPG) approach in computational mechanics," Computational Mechanics, vol. 22, no. 2, pp. 117-127, 1998.

[12] M. Peng, P. Liu, and Y. Cheng, "The complex variable elementfree Galerkin (CVEFG) method for two-dimensional elasticity problems," International Journal of Applied Mechanics, vol. 1, no. 2, pp. 367-385, 2009.

[13] M. Peng, D. Li, and Y. Cheng, "The complex variable elementfree Galerkin (CVEFG) method for elasto-plasticity problems," Engineering Structures, vol. 33, no. 1, pp. 127-135, 2011.

[14] Y. Cheng, J. Wang, and R. Li, “The complex variable elementfree galerkin (CVEFG) method for two-dimensional elastodynamics problems," International Journal of Applied Mechanics, vol. 4, no. 4, Article ID 1250042, 2012.

[15] S. C. Li, S. C. Li, and Y. M. Cheng, "Enriched meshless manifold method for two-dimensional crack modeling," Theoretical and Applied Fracture Mechanics, vol. 44, no. 3, pp. 234-248, 2005.

[16] H. Gao and Y. Cheng, "A complex variable meshless manifold method for fracture problems," International Journal of Computational Methods, vol. 7, no. 1, pp. 55-81, 2010. 
[17] R. J. Cheng and K. M. Liew, "Analyzing two-dimensional sineGordon equation with the mesh-free reproducing kernel particle Ritz method," Computer Methods in Applied Mechanics and Engineering, vol. 245-246, pp. 132-143, 2012.

[18] Y. Cheng and M. Peng, "Boundary element-free method for elastodynamics," Science in China G: Physics Mechanics and Astronomy, vol. 48, no. 6, pp. 641-657, 2005.

[19] K. M. Liew, Y. Cheng, and S. Kitipornchai, "Boundary elementfree method (BEFM) and its application to two-dimensional elasticity problems," International Journal for Numerical Methods in Engineering, vol. 65, no. 8, pp. 1310-1332, 2006.

[20] M. Peng and Y. Cheng, "A boundary element-free method (BEFM) for two-dimensional potential problems," Engineering Analysis with Boundary Elements, vol. 33, no. 1, pp. 77-82, 2009.

[21] S. N. Atluri, J. Sladek, V. Sladek, and T. Zhu, "Local boundary integral equation (LBIE) and it's meshless implementation for linear elasticity," Computational Mechanics, vol. 25, no. 2, pp. 180-198, 2000.

[22] B. Dai and Y. Cheng, "An improved local boundary integral equation method for two-dimensional potential problems," International Journal of Applied Mechanics, vol. 2, no. 2, pp. 421436, 2010

[23] Y. Xie Mukherjee and S. Mukherjee, "The boundary node method for potential problems," International Journal for Numerical Methods in Engineering, vol. 40, no. 5, pp. 797-815, 1997.

[24] D. Levin, "The approximation power of moving least-squares," Mathematics of Computation, vol. 67, no. 224, pp. 1517-1531, 1998.

[25] M. G. Armentano, "Error estimates in Sobolev spaces for moving least square approximations," SIAM Journal on Numerical Analysis, vol. 39, no. 1, pp. 38-51, 2002.

[26] C. Zuppa, "Error estimates for moving least square approximations," Bulletin of the Brazilian Mathematical Society, vol. 34, no. 2, pp. 231-249, 2003.

[27] R. Cheng and Y. Cheng, "Error estimates for the finite point method," Applied Numerical Mathematics, vol. 58, no. 6, pp. 884-898, 2008.

[28] X. Li and J. Zhu, "A Galerkin boundary node method and its convergence analysis," Journal of Computational and Applied Mathematics, vol. 230, no. 1, pp. 314-328, 2009.

[29] H. Ren, J. Cheng, and A. Huang, "The complex variable interpolating moving least-squares method," Applied Mathematics and Computation, vol. 219, no. 4, pp. 1724-1736, 2012.

[30] L. Gavete, M. L. Gavete, B. Alonso, and A. J. Martín, "A posteriori error approximation in EFG method," International Journal for Numerical Methods in Engineering, vol. 58, no. 15, pp. 2239-2263, 2003.

[31] R. Rossi and M. K. Alves, "Recovery based error estimation and adaptivity applied to a modified element-free Galerkin method," Computational Mechanics, vol. 33, no. 3, pp. 194-205, 2004.

[32] R.-J. Cheng and Y.-M. Cheng, "Error estimates of element-free Galerkin method for potential problems," Acta Physica Sinica, vol. 57, no. 10, pp. 6037-6046, 2008.

[33] R.-J. Cheng and Y.-M. Cheng, "Error estimate of element-free Galerkin method for elasticity," Acta Physica Sinica, vol. 60, no. 7, Article ID 070206, 2011.

[34] R. Hongping, C. Yumin, and Z. Wu, "An interpolating boundary element-free method (IBEFM) for elasticity problems," Science China Physics, Mechanics and Astronomy, vol. 53, no. 4, pp. 758766, 2010. 


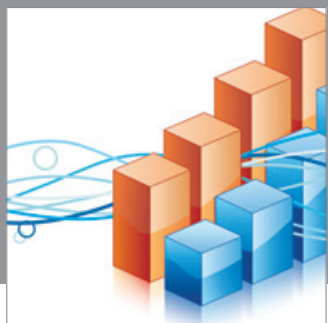

Advances in

Operations Research

mansans

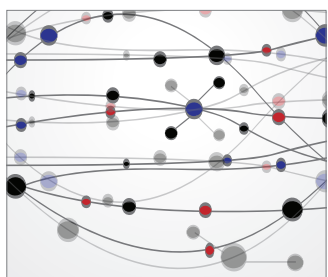

The Scientific World Journal
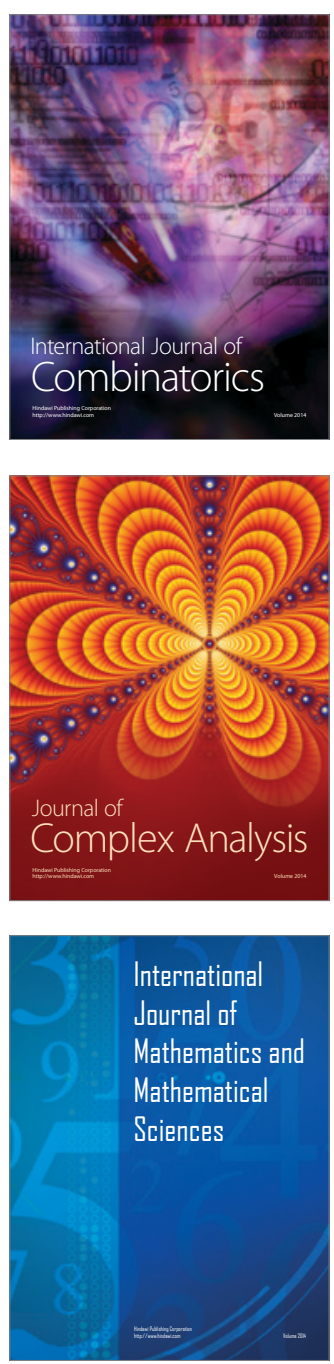
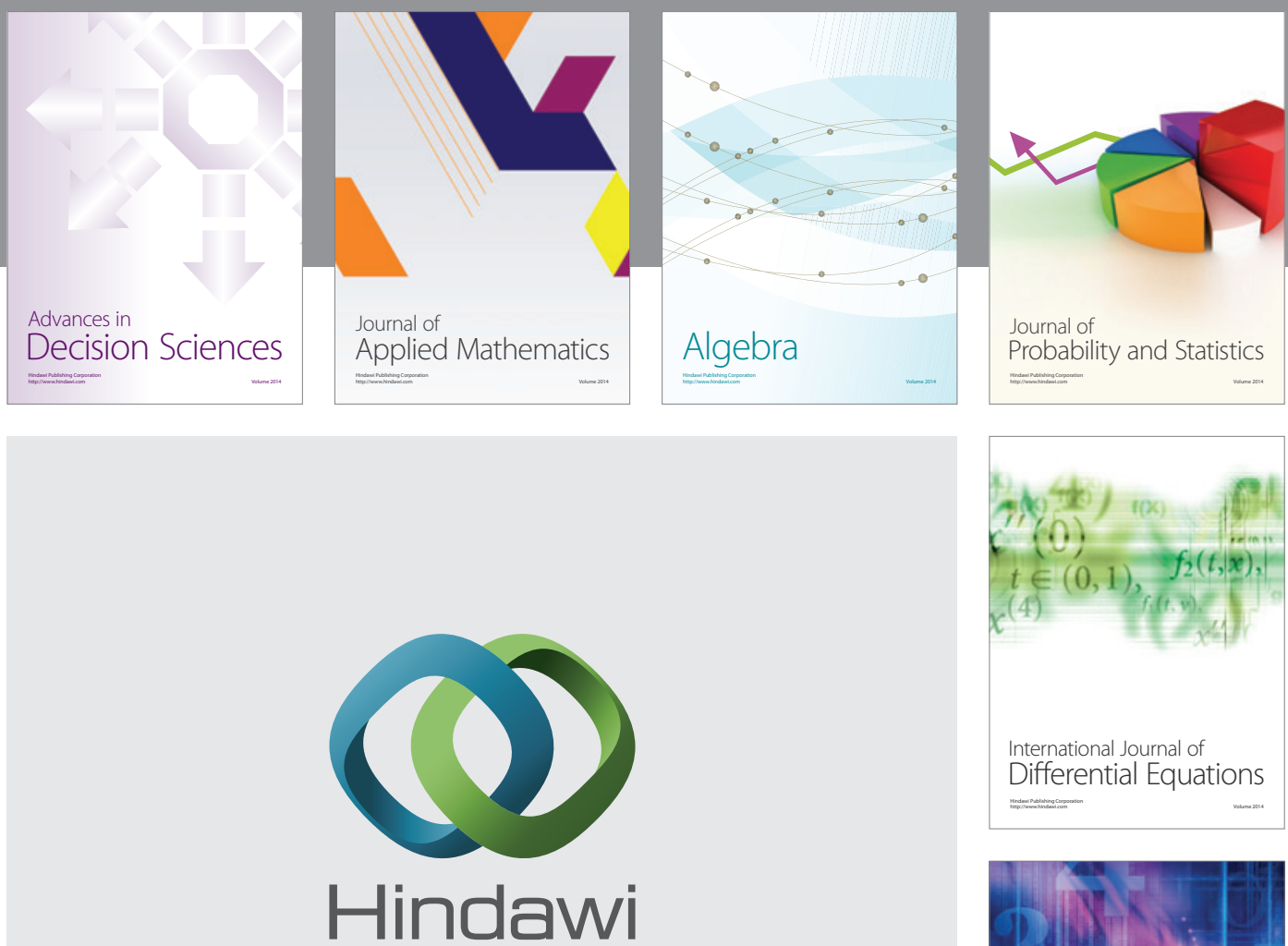

Submit your manuscripts at http://www.hindawi.com
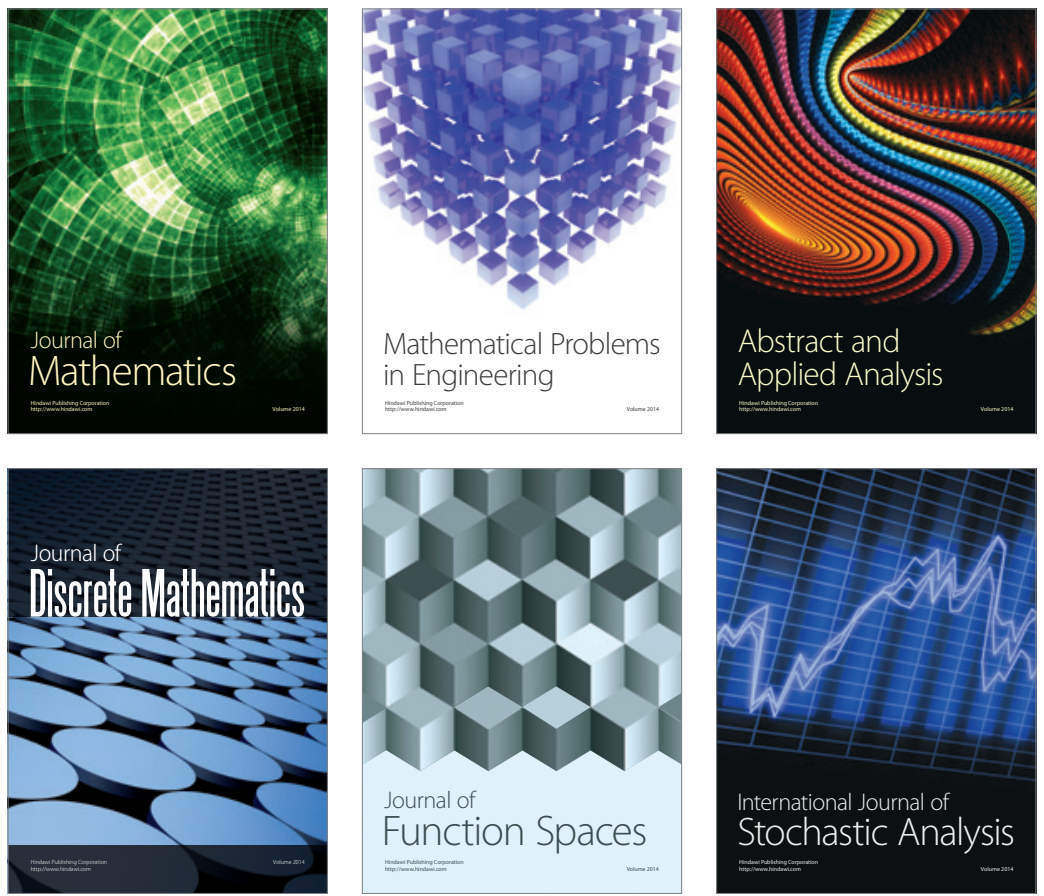

Journal of

Function Spaces

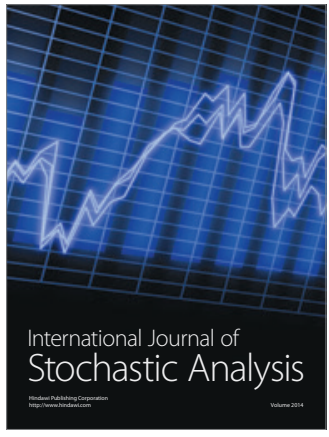

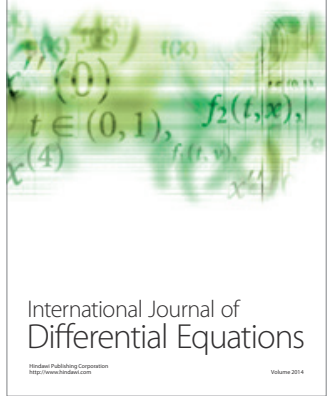
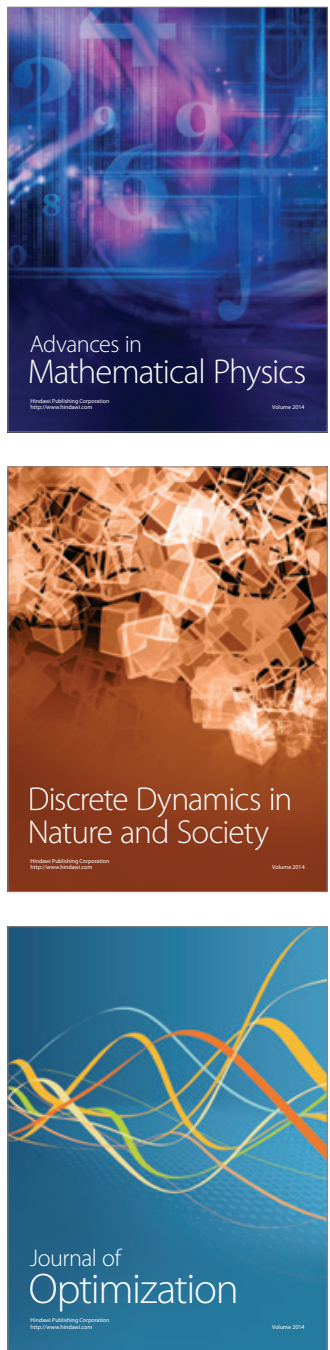